Research Article

\title{
A Joint Optimization Model of Production Scheduling and Maintenance Based on Data Driven for a Parallel-Series Production Line
}

\author{
Kai Zhu \\ Business School, University of Shanghai for Science and Technology, 516 Jungong Road, Shanghai 200093, China \\ Correspondence should be addressed to Kai Zhu; ishzhukai@usst.edu.cn
}

Received 2 August 2021; Accepted 27 August 2021; Published 27 September 2021

Academic Editor: Ching-Feng Wen

Copyright (c) 2021 Kai Zhu. This is an open access article distributed under the Creative Commons Attribution License, which permits unrestricted use, distribution, and reproduction in any medium, provided the original work is properly cited.

\begin{abstract}
The maintenance of a production line is becoming more important with the development of demanding higher operational efficiency and safety in industrial system. However, a production line often operates under dynamically operational and environmental conditions and the production scheduling is also a very important factor for the maintenance of a production line. First, this paper proposes an integrated data-driven model that coordinates maintenance planning decisions with production scheduling decisions to solve the problem of scheduling and maintenance planning for a parallel-series production line. The degradation information is considered, and the total cost is to be minimized in the proposed model. Also, the total cost is related with production process and maintenance considering reliability of equipment. Then, in order to better describe the relationship between production and maintenance, the accumulative processing time of equipment is used as the input of its failure function. Also, an ability factor is developed to control its reduced level by adopting preventive maintenance. Finally, a case study is used to demonstrate the implementation and potential applications of the proposed model. The long-term wear test experiments are conducted at a research laboratory facility of Shanghai Pangyuan Machinery Co., Ltd. The result proves that the proposed method is feasible and efficient to solve the joint decision-making problem for a parallel-series production line with multivariety and small batch production. The proposed model in this paper is suitable for semiconductor manufacturing.
\end{abstract}

\section{Introduction}

Production scheduling and maintenance planning are among the most common and significant problems faced by the manufacturing industries [1-5]. Production scheduling problems generally involve the assignment of jobs or operations to machines, while maintenance actions are carried out to retain a system or to restore it to an acceptable operating condition. Despite the trade-offs between the two activities, they are typically planned and executed independently in real manufacturing settings even if manufacturing productivity can be improved by optimizing both production scheduling and maintenance planning decisions simultaneously.

In a real production line, one machine may fail due to its degradation and usage. The consequent repair and replacement will make machines unavailable, which disorders production scheduling. Hence, how to schedule maintenance planning to keep machines in good operating condition and with high reliability and further make production scheduling based on maintenance has become a vital issue for the achievement of manufacturing [6-10].

For a parallel-series production line, the problem of single machine availability captures a growing interest in a variety of areas [11-14]. Pan et al. [15] proposed a scheduling model for single machine system incorporating production scheduling and machine maintenance, so as to maximize the machine's availability. A heuristic algorithm was proposed to optimize the integrated model of production scheduling and PM [16]. Subsequently, a method for joint production scheduling problem by considering multiresources and preventive maintenance was proposed [17] and its validity was proved. This model has been used for a repairable system [18], and an improved genetic algorithm was used to solve 
the joint problem for a single machine, and joint decisionmaking policy could obtain better result than independent policy [19]. Other areas of application of this problem, including production and assembly lines, communication and radar systems, signal processing, and surveillance, are discussed by Mirabedini et al. [20, 21].

Most maintenance scheduling (MS) problems appearing in the literature involve a rather simple maintenance plan, and the joint optimization of a multiple equipment system can be more complex. Berrichi et al. [22] developed the problem of a parallel system and used an ant colony algorithm to solve it. Also, Moradi et al. [23] researched a flexible job-shop problem and integrated production scheduling and fixed interval preventive maintenance. A dynamic preventive maintenance for a multicomponent system was integrated into a production scheduling [24]. An improved genetic algorithm based on mixed coding was used to solve this integrated model [25]. They formulated a Markov decision process model to determine the maintenance plan and develop sufficient conditions guaranteeing its monotonicity in both machine condition and demand [26, 27]. A multiobjective approach for parallel machines was proposed to allow decision-makers to find compromise solution between production scheduling and maintenance planning: minimizing both maximum completion time and total maintenance cost $[28,29]$. Chang [30] presented a service-oriented dynamic multilevel predictive maintenance grouping strategy. Yan et al. [31] presented a joint optimization method for both buffer stocks control and preventive maintenance inspection interval. Celen and Djurdjanovic [32] proposed a decision-making method based on a partially observable Markov decision process to deal with interactions between maintenance and production operations in flexible manufacturing systems (FMSs). For a parallelseries production line with multivariety and small batch production, Wu et al. [33] considered joint production and maintenance scheduling of a multiproduct batch chemical manufacturing plant by an industrial case study. Li [34] optimized the setup time of the product to achieve the purpose of improving the time efficiency for the multiproduct production system in the factories worldwide. Xia et al. [35] developed a fleet maintenance cost saving (FMCS) policy to optimize condition-based opportunistic maintenance and reduce the total maintenance cost efficiently.

In this paper, we develop an integrated data-driven model incorporating production scheduling and maintenance planning for a parallel-series production line with multivariety and small batch production. The existing literature often ignores the influence of machine degradation on production scheduling, and only few researchers studied processing time model based on machine's usage. However, machine's usage cannot describe its real health condition. Thus, the motivations and contributions of the paper include that processing time model can be improved, an integrated mathematical model considering scheduling and maintenance is proposed, and a parallel-series production line with multivariety and small batch production is considered. In this paper, first, the degradation information of the machine based on Weibull distribution is integrated into the proposed model. The joint objective function considers production cost, preventive maintenance cost, minor repair cost for unexpected failures, and replacement cost. Second, in order to better describe the relationship between production and maintenance, the accumulative processing time of equipment is used as the input of its failure function, and the failure rate is the direct factor affecting maintenance planning. The planning output will directly influence the accumulative processing time of equipment and then affect the failure function of equipment. Moreover, the actual maximum available processing time of each cycle equals the maximum available processing time minus the maintenance time. Then, in order to better describe the maintenance effect, the accumulative processing time of equipment is described as an index to reflect the maintenance effect. The maintenance effect can partly reduce the accumulative processing time. Moreover, an ability factor is developed to control its reduced level by adopting preventive maintenance and the replacement can reduce the accumulative processing time to 0 . Finally, a case study demonstrates the potential applications of the proposed model. The optimal maintenance planning and assignment of each job can be obtained. By comparing these solutions with the results obtained from conducting the preventive maintenance planning and job scheduling problems independently, the result indicates that the proposed method is feasible and efficient to solve the joint decision-making problem for a parallel-series production line with multivariety and small batch production.

This paper is organized as follows. Section 2 describes the parameters of the proposed models. Section 3 focuses on the development of the data-driven production scheduling approach for the parallel-series production line. Section 4 focuses on the development of the datadriven maintenance planning approach for the parallelseries production line. Section 5 presents the joint optimization data-driven model of production scheduling and maintenance planning for the parallel-series production line with multivariety and small batch production. A numerical example is introduced in Section 6, and some results are discussed. Section 7 draws some conclusions from this work.

\section{Notation}

In this paper, the parameters are described as follows:

$N$ : the number of production cycles during the planning period

$K$ : types of products

$M$ : the number of equipment in the production line

$U_{\max }$ : maximum available processing time of one production cycle

$s c_{k}$ : setup cost of product $k, k=1,2, \ldots, K$

$p c_{k}$ : production cost of product $k$ per unit, $k=1,2, \ldots, K$

$h_{k}$ : inventory cost of product $k$ per unit, $k=1,2, \ldots, K$

$a_{k}$ : processing time of product $k, k=1,2, \ldots, K$ 
$s_{k i}$ : inventory of $k$ at the end of $i$-th cycle, $k=1,2, \ldots, K$ and $i=1,2, \ldots, N$

$d_{k i}$ : demand for $k$ in $i$-th cycle, $k=1,2, \ldots, K$ and $i=1$, $2, \ldots, N$

$c_{m}$ : repair preparation cost for one time

$A_{j}$ : preventive maintenance cost of equipment $j$ for one time, $j=1,2, \ldots, M$

$R_{j}$ : replacement cost of equipment $j$ for one time, $j=1$, $2, \ldots, M$

$F_{j}$ : minor repair cost of equipment $j$ for one time, $j=1$, $2, \ldots, M$

$E\left(B_{i j}\right)$ : expectation of the number of failures of equipment $j$ occurred in $i$-th cycle, $i=1,2, \ldots, N$ and $j=1,2, \ldots, M$

$z_{i j}$ : accumulative processing time of equipment $j$ from initial using time to the end of $i$-th cycle, $i=1,2, \ldots, N$ and $j=1,2, \ldots, M$

$\lambda_{j 0}(t)$ : failure rate function of equipment $j$ under the maximum production ability, $j=1,2, \ldots, M$

$t_{p}$ : preventive maintenance time

$t_{r}$ : replacement time of equipment, $t_{r}>t_{p}$

$b_{j}$ : ability factor of preventive maintenance and it reflects the ability reduced accumulative processing time of equipment $j$ by preventive maintenance, $j=1,2, \ldots, M$

$\lambda_{i j}(t)$ : failure rate function of equipment $j$ in $i$-th cycle and it is related with accumulative processing time of equipment $j$ and maximum available processing time, $i=1,2, \ldots, N$ and $j=1,2, \ldots, M$

Variable:

$x_{k i}$ : planning output of product $k$ in $i$-th cycle, $k=1,2, \ldots, K$ and $i=1,2, \ldots, N$

$s t_{k i}$ : binary variable $\left(s t_{k i}=1\right.$ if $k$ is produced in $i$-th cycle; otherwise, $\left.s t_{k i}=0\right), k=1,2, \ldots, K$ and $i=1,2, \ldots, N$ $m_{i j}$ : binary variable, performed preventive maintenance action for equipment $j$ at the beginning of $i$-th cycle, $i=1,2, \ldots, N$ and $j=1,2, \ldots, M$

$r_{i j}$ : binary variable, performed replacement action for equipment $j$ at the beginning of $i$-th cycle, $i=1,2, \ldots, N$ and $j=1,2, \ldots, M$

$C_{1}$ : total production cost

$\mathrm{C}_{2}$ : total maintenance cost

$\mathrm{C}_{3}$ : total cost

\section{Production Scheduling Model}

For a parallel-series production line, which produces $K$ products, a planning period consisting of $N$ production cycles is considered. Assume that the demand of each product is $d_{k i}(k=1,2, \ldots, K ; i=1,2, \ldots, N)$ at the beginning of each production cycle; $d_{k i}$ can be obtained by orders or marketing prediction. The production line will generate a production preparation cost for each production cycle. The enterprises complete all demands after the production line is started. However, inventories can be accumulated by producing large quantities of products at one time, which can lead a larger inventory cost and generate a greater harm to the production line. Thus, the optimal production quantity $x_{k i} \quad(k=1,2, \ldots, K ; i=$ $1,2, \ldots, N)$ at the beginning of each production cycle can be obtained by using the production scheduling model with constraints of production capacity. It can satisfy the demand of each product at the beginning of each cycle, balance the production preparation cost and the inventory cost, and also minimize the total production cost including the production preparation cost, production cost, and inventory cost.

The data-driven production scheduling optimization model for a parallel-series production line with multivariety and small batch production is as follows:

$\operatorname{Min} C_{1}=\sum_{k=1}^{K} \sum_{i=1}^{N}\left(s c_{k} \times s t_{k i}+p c_{k} \times x_{k i}+h_{k} \times s_{k i}\right)$,

s.t.

$$
\begin{aligned}
& \sum_{k=1}^{K} a_{k} \times x_{k i} \leq U_{\max }, \quad i=1,2, \ldots, N, \\
& s_{k(i+1)}=s_{k i}+x_{k(i+1)}-d_{k(i+1)}, \quad k=1,2, \ldots, K, i=1,2, \ldots, N, \\
& s t_{k i}=\left\{\begin{array}{l}
1, \quad \text { if } x_{k i}>0, \quad k=1,2, \ldots, K, i=1,2, \ldots, N, \\
0, \quad \text { if } x_{k i}=0,
\end{array}\right. \\
& 0 \leq x_{k i} \leq \sum_{l=i}^{N} d_{k l}, \quad k=1,2, \ldots, K, i=1,2, \ldots, N, \\
& s_{k 1}=x_{k 1}-d_{k 1}, \quad k=1,2, \ldots, K, \\
& s_{k i} \geq 0,=1,2, \ldots, K, i=1,2, \ldots, N .
\end{aligned}
$$

Equation (1) denotes the objective function of production scheduling model, which is to minimize the total production cost, including production preparation cost, production cost, and inventory cost, during a planning period. Equation (2) is the production capacity constraint, and it denotes that the production time of each production cycle must be no more than its available maximum processing time $U_{\max }$. Equation (3) describes the inventory and reflects the relationship between demand and inventory. The inventory of product $k$ at the end of $(i+1)$-th cycle equals the summation of its inventory at the end of $i$ th cycle plus the planning output $x_{k(i+1)}$ at $(i+1)$-th cycle minus demand $d_{k(i+1)}$ at $(i+1)$-th cycle. Equation (4) denotes the production preparation cost of product $k$ at $i$ th cycle. Also, if product $k$ is produced at $i$-th cycle, then $s t_{k i}$ equals 1, otherwise, 0. Equation (5) is the production capacity constraint of product $k$ at $i$-th cycle. Also, the planning output of the current cycle cannot exceed the 
accumulated demand of the current cycle and the following cycles due to the assumption that inventory after finishing the planning period is 0 . Equation (6) denotes the inventory of product $k$ at the end of the first cycle (assuming that the inventory of product $k$ at the beginning of first cycle is 0). Equation (7) is the nonnegative constraint of inventory.

\section{Maintenance Planning Model}

Equipment can wear out with the increase of production time, and failures can interrupt the production. Thus, maintenance activities need to be performed in order to achieve continuous production. In the current paper, the maintenance activities include nonrepair, preventive maintenance, and replacement. The preventive maintenance can decrease the accumulative processing time of equipment, and the replacement can reset the accumulative processing time of equipment. Moreover, because of some random failures during practical production, minor repair can be performed in order to carry out production and avoid shortage, which makes equipment restore to production status as soon as possible. Thus, minor repair time can be neglected and it cannot influence the accumulative processing time of equipment. The objective of the maintenance planning model is to minimize the total maintenance cost, including maintenance preparation cost, preventive maintenance cost, replacement cost, and minor repair cost. The aim of the model is to choose the optimal maintenance activities for equipment at the beginning of each cycle.

In this section, the data-driven maintenance planning optimization model of the production line with multivariety and small batch production can be obtained as follows:

$$
\begin{array}{ll}
\text { Min } & C_{2}=\sum_{i=1}^{N} c_{m} \times\left(1-\prod_{j=1}^{M}\left(1-m_{i j}\right)\left(1-r_{i j}\right)\right)+\sum_{i=1}^{N} \sum_{j=1}^{M}\left(A_{j} \times m_{i j}+R_{j} \times r_{i j}+F_{j} \times E\left(B_{i j}\right)\right), \\
\text { s.t. } & \\
& m_{i j}+r_{i j} \leq 1, \quad i=1,2, \ldots, N, j=1,2, \ldots, M \\
& m_{i j}, r_{i j} \in\{0,1\}, \quad i=1,2, \ldots, N, j=1,2, \ldots, M
\end{array}
$$

Equation (8) denotes the objective function of maintenance planning model, which is to minimize the total maintenance cost, including maintenance preparation cost, preventive maintenance cost, replacement cost, and minor repair cost, during a planning period. The expected number of random failures at $i$-th cycle is

$$
E\left(B_{i j}\right)=\int_{z_{(i-1) j} \times\left[\left(1-m_{i j}\right)\left(1-r_{i j}\right)+b_{j} \times m_{i j}\right]}^{z_{i j}} \lambda_{j 0}(t) \mathrm{d} t,
$$

where $z_{i j}$ denotes the accumulative processing time of equipment $j$ from the beginning to the end of $i$-th cycle. Equation (9) is the maintenance constraint of equipment and denotes that only one maintenance activity is performed at the beginning of each cycle. If $m_{i j}=0$ and $r_{i j}=0$, there will be nonrepair at the beginning of $i$-th cycle for equipment $j$. If $m_{i j}=1$ and $r_{i j}=0$, preventive maintenance will be performed at the beginning of $i$-th cycle for equipment $j$. If $m_{i j}=0$ and $r_{i j}=1$, replacement will be performed at the beginning of $i$-th cycle for equipment $j$. Equation (10) is the binary variables constraint of maintenance.
The maintenance effect is to decrease the accumulative processing time of equipment. If the accumulative processing time of equipment $j$ at the end of $(i-1)$-th cycle is $z_{(i-1) j}$ and the preventive maintenance is performed for equipment $j$ during the $i$-th cycle, the accumulative processing time of equipment $j$ becomes $b_{j} \times z_{(i-1) j}$. If the replacement is performed for equipment $j$, the accumulative processing time of equipment $j$ will be reset. Thus, at the end of $i$-th cycle, there are three descriptions for $z_{i j}$, including nonrepair, preventive maintenance, and replacement.

$$
z_{i j}= \begin{cases}z_{(i-1) j}+U_{\max }-t_{p} \times m_{i j}-t_{r} \times r_{i j}, & m_{i j}=0, r_{i j}=0, \\ b_{j} \times z_{(i-1) j}+U_{\max }-t_{p} \times m_{i j}-t_{r} \times r_{i j}, & m_{i j}=1, r_{i j}=0, \\ U_{\max }-t_{p} \times m_{i j}-t_{r} \times r_{i j}, & m_{i j}=0, r_{i j}=1 .\end{cases}
$$

Based on equation (12), $z_{i j}$ can be obtained as follows: 


$$
z_{i j}=U_{\max }-\max \left\{\left[1-\prod_{j=1}^{M}\left(1-m_{i j}\right)\right] \times t_{p},\left[1-\prod_{j=1}^{M}\left(1-r_{i j}\right)\right] \times t_{r}\right\}+z_{(i-1) j} \times\left[\left(1-m_{i j}\right)\left(1-r_{i j}\right)+b_{j} \times m_{i j}\right]
$$

where $z_{1 j}=U_{\max }-t_{p} \times m_{1 j}-t_{r} \times r_{1 j}$.

\section{Joint Optimization Model of Production and Maintenance}

A production line possesses the production requirements defined in Section 3 and the failure, replacement, and preventive maintenance characteristics described in Section 4. Therefore, the data-driven joint optimization model is introduced to address this problem due to its excellent performance in fusing multiple features in the process of ranking. Furthermore, assume that products are not pre-empted for preventive maintenance and products interrupted by failure can be resumed after maintenance without any additional time. Both production scheduling and maintenance planning are to minimize the total production/maintenance cost. Thus, it is superior to solve the production scheduling and maintenance planning problems for the production line simultaneously than independently. For obtaining an optimal production planning, decision on whether to perform maintenance activities or not must be made. The integrated problem is more complicated because the production line includes parallel and series equipment simultaneously. Equipment may or may not fail during each production cycle, and maintenance decisions will divide all equipment into three groups at the same time, including nonrepair group, replacement group, and preventive maintenance group, and it can decrease the maintenance cost and increase productivity.

The assumptions for the joint optimization problem are as follows:

(1) In the parallel-series production line, the type of parallel equipment is the same.

(2) The production preparation time at the beginning of each cycle is used to maintain equipment. The time of replacement is longer than preventive maintenance time; thus, the replacement time is described as the maintenance time if the replacement is performed.

(3) The planning output of parallel equipment is equal.

For a production line with multivariety and small batch production, the integrated optimization model of production scheduling and maintenance planning is as follows:

$$
\begin{aligned}
\operatorname{Min} C_{3}= & \sum_{k=1}^{K} \sum_{i=1}^{K}\left(s c_{k} \times s t_{k i}+p c_{k} \times x_{k i}+h_{k} \times s_{k i}\right) \\
& +\sum_{i=1}^{N} c_{m} \times\left(1-\prod_{j=1}^{M}\left(1-m_{i j}\right)\left(1-r_{i j}\right)\right)+\sum_{i=1}^{N} \sum_{j=1}^{M}\left(A_{j} \times m_{i j}+R_{j} \times r_{i j}+F_{j} \times E\left(B_{i j}\right)\right),
\end{aligned}
$$

s.t.

$$
\begin{aligned}
& \sum_{k=1}^{K} a_{k} \times x_{k i} \leq U_{\max }-\max \left\{\left[1-\prod_{j=1}^{M}\left(1-m_{i j}\right)\right] t_{p},\left[1-\prod_{j=1}^{M}\left(1-r_{i j}\right)\right] t_{r}\right\} \\
& s_{k(i+1)}=s_{k i}+x_{k(i+1)}-d_{k(i+1)}, \quad k=1,2, \ldots, K, i=1,2, \ldots, N, \\
& s t_{k i}=\left\{\begin{array}{l}
1, \quad \text { if } x_{k i}>0, \quad k=1,2, \ldots, K, i=1,2, \ldots, N, \\
0, \quad \text { if } x_{k i}=0,
\end{array}\right. \\
& m_{i j}+r_{i j} \leq 1, \quad i=1,2, \ldots, N, j=1,2, \ldots, M, \\
& 0 \leq x_{k i} \leq \sum_{i=1}^{N} d_{k l}, \quad k=1,2, \ldots, K, i=1,2, \ldots, N, \\
& s_{k 1}=x_{k 1}-d_{k 1}, \quad k=1,2, \ldots, K, \\
& s_{k i} \geq 0, \quad k=1,2, \ldots, K, i=1,2, \ldots, N, \\
& m_{i j}, r_{i j} \in\{0,1\}, \quad i=1,2, \ldots, N, j=1,2, \ldots, M .
\end{aligned}
$$


Equation (14) denotes the objective function of the integrated optimization model, which is to minimize the total production cost, including production preparation cost, production cost and inventory cost, and the total maintenance cost, including maintenance preparation cost, preventive maintenance cost, replacement cost, and minor repair cost, during a planning period. Equation (15) is the production capacity constraint and denotes that the maximum available processing time equals the maximum available processing time $U_{\max }$ minus the maintenance activities time. Equation (16) describes the inventory, and it reflects the relationship among demand, inventory, and output. Also, the inventory of product $k$ at the end of $(i+1)$-th cycle equals the summation of its inventory at the end of $i$-th cycle and the planning output $x_{k(i+1)}$ minus demand $d_{k(i+1)}$ during $(i+1)$-th cycle. Equation (17) denotes the production preparation cost of product $k$ at $i$ th cycle. Also, if product $k$ is produced at $i$-th cycle, $s t_{k i}$ equals 1, otherwise 0. Equation (18) is the maintenance constraint, and it denotes that only one maintenance activity is performed at the beginning of each cycle. Equation (19) is the output constraint of product $k$ at $i$-th cycle. Also, the planning output of the current cycle cannot exceed the accumulated demand of the current cycle and the following cycles because the output depends on demand. Equation (20) denotes the initial value of the inventory. Equation (21) is the nonnegative constraint of inventory. Equation (22) is the binary variable constraint of maintenance.

$E\left(B_{i j}\right)=\int_{0}^{U_{\max }} \lambda_{j 0}(t) \mathrm{d} t$ describes the expectation of random failures of equipment $j$ in $i$-th cycle. The accumulative processing time of equipment reflects the influence of production on maintenance. Thus, the failure rate function of equipment $j$ in $i$-th cycle can be expressed as follows:

$$
\lambda_{i j}(t)=g_{j}\left(x_{i}\right) \times \lambda_{j 0}(t)=\frac{z_{i j}\left(x_{i}\right)}{U_{\max }} \times \lambda_{j 0}(t),
$$

where $z_{i j}\left(x_{i}\right)$ denotes the accumulative processing time of all products completed by equipment $j$ from initial production to the end of $i$-th cycle and $\lambda_{j 0}(t)$ is the failure rate function of equipment $j$ under the maximum production capacity.

For a production line, the production is completed by using multiequipment. Also, the production output of each equipment in the production line is equal, so the output of product $k$ completed on each equipment is $x_{k i} / n$, where $n$ denotes the number of equipment. The accumulative processing time of equipment in the parallel process can be described as follows:

$$
z_{i j}= \begin{cases}z_{(i-1) j}+\sum_{k=1}^{K} a_{k} \times \frac{x_{k i}}{2}, & m_{i}=0, r_{i}=0, \\ b \times z_{(i-1) j}+\sum_{k=1}^{K} a_{k} \times \frac{x_{k i}}{2}, & m_{i}=1, r_{i}=0, \\ \sum_{k=1}^{K} a_{k} \times \frac{x_{k i}}{2}, & m_{i}=0, r_{i}=1 .\end{cases}
$$

Equation (24) denotes the accumulative processing time of equipment $j$ at the end of $i$-th cycle after performing nonrepair, preventive maintenance, and replacement at the beginning of $i$-th cycle, respectively. The three parts of equation (24) can be combined as follows:

$$
z_{i j}=\left[\left(1-m_{i j}\right)\left(1-r_{i j}\right)+b_{j} \times m_{i j}\right] \times z_{(i-1) j}+\sum_{k=1}^{K} a_{k} \times \frac{x_{k i}}{2},
$$

where $z_{1 j}=\sum_{k=1}^{K} a_{k} \times x_{k 1} / 2$.

For the series equipment in the production line, the production output of each equipment may not be equal. Thus, its accumulative processing time can be obtained as follows:

$$
z_{i j}=\left[\left(1-m_{i j}\right)\left(1-r_{i j}\right)+b_{j} \times m_{i j}\right] \times z_{(i-1) j}+\sum_{k=1}^{K} a_{k} \times x_{k i} .
$$

\section{Case Study}

6.1. Experimental Description. In this numerical example, the long-term wear test experiments were conducted at a research laboratory facility (Shanghai Pangyuan Machinery Co., Ltd.). In the test experiments, one pump was worn by running it using oil containing dust. The degradation stages in this hydraulic pump wear test case study correspond to different stages of flow loss in the pump. As the flow rate of a pump clearly indicates pump's health state, the degradation stages corresponding to different degrees of flow loss in a pump were defined as the health states of the pump in the test.

The hydraulic pump needs to produce 2 types of products $(K=2)$, the work of the hydraulic pump (system) needs 5 equipment to cooperate with each other $(M=5)$, and the planning period consists of 8 production cycles $(N=8)$. Thus, maintenance optimization is performed to the 5 equipment of the hydraulic pump. The health of the hydraulic pump will be worn gradually with the increase of time in the process of operations. In order to maintain its normal work and avoid high cost and risk, the pump needs to perform maintenance activities. Moreover, the replacement can be performed with the increase of production cost and maintenance cost. The total cost of both production and maintenance is described as the optimization objective to make the maintenance scheduling strategy. Figure 1 shows the schematic diagram of the relationship among 5 equipment. Equipment 1 is denoted as $M_{1}$. Also, all of the models are coded in Visual C\# and ran on a personal computer with a 2.10 GHzn2 CPU and 4.0 GB RAM. Moreover, the parameters used for the data-driven method are detailed in Tables 1-3.

\subsection{Data Preparation}

6.2.1. Production Cost. For two types of products, the production time per unit is 0.06 and $0.01 \quad\left(a_{1}=0.06\right.$ and $\left.a_{2}=0.01\right)$, respectively. The related costs and demand of each production cycle are given in Tables 1 and 2, respectively. 


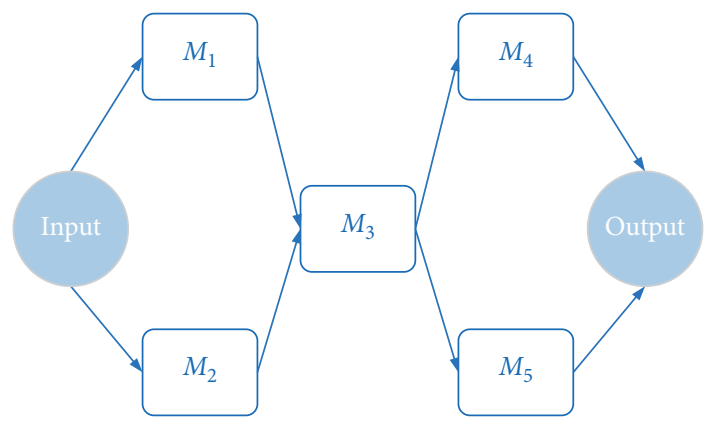

FIGURE 1: The relationship among five machines.

6.2.2. Maintenance Cost. In the process of maintenance optimization, Weibull distribution is used to describe the failure rate and obtain degradation information of the production line. $\lambda_{j}(t)=\left(\beta_{j} / \eta_{j}\right) \times\left(t / \eta_{j}\right)^{\beta_{j}-1}$. Its scale and shape parameters corresponding to different equipment are different. The Weibull parameter values can be calculated based on pump's failure data information from the literature [36] and maximum likelihood estimation [37] (see Table 3). Moreover, the preventive maintenance cost, replacement cost, minor repair cost, and ability factor of preventive maintenance for each machine are also given in Table 3.

6.2.3. Other Parameters. The other parameters are as follows:

$$
\begin{aligned}
c_{m} & =10, \\
t_{p} & =0.9, \\
t_{r} & =2.7, \\
U_{\max } & =30 .
\end{aligned}
$$

Thus, equation (14) is used to combine the data as the input for the proposed data-driven method.

6.3. Result Analysis. In the case study, Visual C\# is used to solve the joint optimization problem between production scheduling and maintenance. The minimal total cost for 8 production cycles of a parallel-series production line can be obtained $\left(\mathrm{C}_{\min }=11206.73\right)$. The optimal maintenance planning can be shown in Table 4 .

It can be seen in Table 4 that equipments 1 and 2 will perform preventive maintenance at the beginning of cycles 2 and 5, respectively. Equipment 4 and 5 will perform preventive maintenance in cycles 3 and 5, respectively. Because of the lower replacement cost, the optimal maintenance planning of equipment 3 will perform replacement at the beginning of cycle 5 . Thus, the optimal maintenance planning for each machine of the parallel-series production line is different due to maintenance cost and degradation of equipment. For industries, health status, failure, and maintenance status of equipment need to be carefully recorded to provide data for production scheduling and maintenance.

In Table 5, the optimal production scheduling is given. Because of the conflict between production preparation cost and inventory cost, the mutual influence between production scheduling and maintenance planning, the optimal planning output of each production cycle is not the actual demand. If these types of data are obtainable in the practical application, they can also be introduced into the feature of scheduling and maintenance in the proposed datadriven method.

6.4. Maintenance Scheduling Strategy Comparison. In order to analyze the performance of the integrated model, it can be compared with the production scheduling model and maintenance planning model by considering the influence of maximum available processing time $U_{\max }$ and the number of production cycles $N$. The total cost of the three models can be computed based on the different $U_{\max }$ and $N$, respectively. Moreover, the difference diff is described as the summation of the optimal production cost and the optimal maintenance cost minus the optimal total cost of the integrated model based on different $U_{\max }$, and it can be found in Table 6 .

Table 6 shows that the integrated model has a better performance for saving cost based on different maximum available processing time $U_{\max }$ and the diff is proportional to $U_{\max }$. Similarly, the integrated model can better decrease the total cost with the increase of the production capacity.

Table 7 shows the diff that the summation of the optimal production cost and the optimal maintenance cost minus the optimal total cost of the integrated model based on different production cycles $N$. Also, the integrated model has a better performance for saving cost than the cost summation of production scheduling model and maintenance planning model. The diff is most when the number of production cycle for one planning period is 5 , and it decreases gradually with the increase of the number of production cycles. It indicates that the production cycle for one planning period needs to be limited in industries based on joint optimization results. In this case, the optimal production cycle for one planning period is 5 . The integrated model cannot obtain the best performance.

In order to directly describe the superiority of $U_{\max }$ and $N$ to the integrated model, Figure 2 is used to analyze the change of diff. In Figure 2, the curve reflects the change of diff with the increase of $U_{\max }$ from the bottom to top. The diff is proportional to $U_{\max }$ for the certain $N$. The diff increases and then decreases with the change of the number of $N$. Moreover, there is always one $N^{*}$ so that the integrated model can obtain best performance.

Nowadays, periodic maintenance, defined as significant activities carried out regularly to maintain condition or operational status of a parallel-series production line, is a common maintenance strategy. The periodic maintenance includes periodic inspections, periodic repairs, and preventive maintenance. In this paper, periodic maintenance aims to obtain the optimal maintenance strategies in one life cycle of a parallel-series production line [11]. The integrated model can be compared with the periodic maintenance model integrated production by considering the influence of maximum available processing time $U_{\max }$ and the number of production cycles $N$. The total cost of the two models can be computed based on the different $U_{\max }$ and $N$. 
TABLE 1: The related costs of products.

\begin{tabular}{lccc}
\hline Product & $s c_{k}$ & $p c_{k}$ & \\
\hline$K=1$ & 90 & 3 & $h_{k}$ \\
$K=2$ & 120 & 4 & 0.1 \\
\hline
\end{tabular}

TABLE 2: The demand of products.

\begin{tabular}{lcccccccc}
\hline$i$ & 1 & 2 & 3 & 4 & 5 & 6 & 7 & 8 \\
\hline$d_{2 i}$ & 206 & 198 & 184 & 217 & 183 & 105 & 140 & 237 \\
$d_{2 i}$ & 116 & 130 & 160 & 135 & 123 & 168 & 160 & 115 \\
\hline
\end{tabular}

TABLE 3: The related maintenance parameters.

\begin{tabular}{lccccc}
\hline$j$ & 1 & 2 & 3 & 4 & 50 \\
\hline$\eta_{j}$ & 50 & 50 & 58 & 60 \\
$\beta_{j}$ & 2 & 2 & 2 & 2 & 25 \\
$A_{j}$ & 15 & 15 & 20 & 2 & 25 \\
$R_{j}$ & 1000 & 1000 & 100 & 70 & 2000 \\
$F_{j}$ & 60 & 60 & 0.7 & 0.6 & 0.6 \\
$b_{j}$ & 0.5 & 0.5 & & 0.6 \\
\hline
\end{tabular}

TABLE 4: The optimal maintenance planning.

\begin{tabular}{llcccccc}
\hline$i$ & 1 & 2 & 3 & 4 & 5 & 6 & 7 \\
\hline$m_{i 1}, r_{i 1}$ & 0 & $m_{12}=1$ & 0 & 0 & $m_{15}=1$ & 0 & 0 \\
$m_{i 2}, r_{i 2}$ & 0 & $m_{22}=1$ & 0 & 0 & $m_{25}=1$ & 0 & 0 \\
$m_{i 3}, r_{i 3}$ & 0 & 0 & 0 & 0 & $r_{35}=1$ & 0 & 0 \\
$m_{i 4}, r_{i 4}$ & 0 & 0 & 0 & 0 & $m_{45}=1$ & 0 & 0 \\
$m_{i 5}, r_{i 5}$ & 0 & 0 & $m_{53}=1$ & 0 & $m_{55}=1$ & 0 & 0 \\
\hline
\end{tabular}

TABLE 5: The optimal production scheduling.

\begin{tabular}{lcccccccc}
\hline$i$ & 1 & 2 & 3 & 4 & 5 & 6 & 7 & 8 \\
\hline$x_{2 i}$ & 206 & 198 & 184 & 400 & 0 & 105 & 140 & 237 \\
$x_{2 i}$ & 116 & 290 & 0 & 135 & 123 & 168 & 160 & 115 \\
\hline
\end{tabular}

TABLE 6: The difference value between the independent model and integrated model $(N=4)$.

\begin{tabular}{|c|c|c|c|c|c|}
\hline \multirow{2}{*}{$U_{\max }$} & \multicolumn{3}{|c|}{ Production scheduling model and maintenance planning model } & \multirow{2}{*}{ The total cost of the integrated model } & \multirow{2}{*}{ diff } \\
\hline & The total maintenance cost $\left(C_{2}\right)$ & The total production cost $\left(C_{1}\right)$ & $C_{1}+C_{2}$ & & \\
\hline 30 & 700.69 & 5091.50 & 5792.19 & 5398.97 & 393.22 \\
\hline 35 & 863.00 & 5091.50 & 5954.50 & 5356.03 & 598.47 \\
\hline 40 & 1045.57 & 5091.50 & 6137.07 & 5316.08 & 820.47 \\
\hline 45 & 1252.97 & 5091.50 & 6344.47 & 5291.03 & 1053.44 \\
\hline 50 & 1475.00 & 5091.50 & 6566.50 & 5272.59 & 1293.91 \\
\hline 55 & 1719.61 & 5091.70 & 6801.31 & 5257.50 & 1543.81 \\
\hline 60 & 1937.96 & 5091.70 & 7019.66 & 5244.92 & 1774.74 \\
\hline
\end{tabular}

Table 8 shows that the integrated model has a better performance for saving cost based on different maximum available processing time $U_{\max }$, and the diff is proportional to $U_{\max }$. Similarly, the integrated model can better decrease the total cost with the increase of the production capacity.
Table 9 shows the diff that the optimal maintenance cost of periodic maintenance model integrated production minus the optimal total cost of the integrated model based on different production cycles $N$. Also, the integrated model has a better performance for saving cost. 
TABLE 7: The difference value between the independent model and integrated model $\left(U_{\max }=30\right)$.

\begin{tabular}{|c|c|c|c|c|c|}
\hline \multirow{2}{*}{$N$} & \multicolumn{3}{|c|}{ Production scheduling model and maintenance planning model } & \multirow{2}{*}{ The total cost of the integrated model } & \multirow{2}{*}{ diff } \\
\hline & The total maintenance cost $\left(C_{2}\right)$ & The total production cost $\left(C_{1}\right)$ & $C_{1}+C_{2}$ & & \\
\hline 1 & 94.43 & 1292.00 & 1386.43 & 1313.28 & 73.15 \\
\hline 2 & 335.58 & 2451.80 & 2787.38 & 2535.84 & 251.54 \\
\hline 3 & 533.81 & 3796.40 & 4330.21 & 3946.07 & 384.14 \\
\hline 4 & 700.69 & 5091.50 & 5792.19 & 5398.97 & 393.22 \\
\hline 5 & 847.72 & 6268.90 & 7116.62 & 6635.97 & 480.65 \\
\hline 6 & 981.81 & 7350.80 & 8332.61 & 8238.29 & 94.32 \\
\hline 7 & 1105.14 & 8511.20 & 9616.34 & 9598.67 & 17.67 \\
\hline 8 & 1220.43 & 9813.90 & 11034.33 & 11026.73 & 7.6 \\
\hline
\end{tabular}

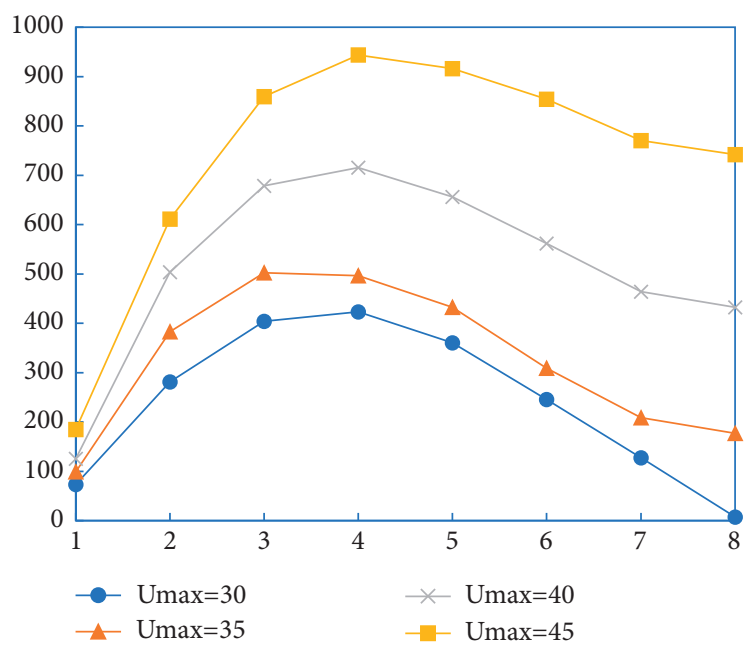

FIgURE 2: The influence of $U_{\max }$ and $N$ on diff.

TABLE 8: The difference value between the periodic maintenance model integrated and integrated model $(N=4)$.

\begin{tabular}{lccc}
\hline$U_{\max }$ & Periodic maintenance model integrated production & The total cost of the integrated model & diff \\
\hline 30 & 5673.23 & 5398.97 & 274.26 \\
35 & 5779.15 & 5356.03 & 423.12 \\
40 & 5836.78 & 5316.08 & 520.7 \\
45 & 6091.56 & 5291.03 & 800.53 \\
50 & 6266.71 & 5272.59 & 994.12 \\
55 & 6535.81 & 5257.5 & 1278.31 \\
60 & 6709.6 & 5244.92 & 1464.68 \\
\hline
\end{tabular}

TABLE 9: The difference value between the periodic maintenance model integrated and integrated model $\left(U_{\max }=30\right)$.

\begin{tabular}{lccc}
\hline$N$ & Periodic maintenance model integrated production & The total cost of the integrated model & diff \\
\hline 1 & 1367.34 & 1313.28 & 54.06 \\
2 & 2616.89 & 2535.84 & 81.05 \\
3 & 4120.97 & 3946.07 & 174.9 \\
4 & 5563.61 & 5398.97 & 164.64 \\
5 & 6998.15 & 6635.97 & 362.18 \\
6 & 8301.66 & 8238.29 & 63.37 \\
7 & 9615.04 & 9598.67 & 16.37 \\
8 & 11031.16 & 11026.73 & 4.43 \\
\hline
\end{tabular}

\section{Conclusions}

Both production scheduling and maintenance planning play important roles in the manufacturing industries. In order to optimize the overall performance of the manufacturing system, it is necessary for enterprises to integrate production scheduling and maintenance planning together.

This paper emphasizes the need of an integrated model for a parallel-series production line with multivariety and small batch production. The objective is to minimize the 
total cost, including production cost and maintenance cost. Also, the total cost is related with production process and maintenance considering reliability of equipment, including preventive maintenance, minor repair for the unexpected failures, and replacement. Moreover, the importance of different equipment is considered in the proposed model. Finally, we investigate the value of integrating production scheduling with maintenance planning by conducting an extensive experimental study to form the input of the datadriven method for multivariety and small batch scheduling problems. From the experiment results, it can be found that the proposed method is superior to the independent production scheduling model and maintenance planning model in terms of both feasibility and efficiency for a parallel-series production line with multivariety and small batch production.

The long-term wear test experiments are conducted at a research laboratory facility of Shanghai Pangyuan Machinery Co., Ltd. Industrial implementation and demonstration of the newly proposed methods in a real factory environment is still maintained, and the primary effect can be obtained. The proposed model in this paper is suitable for semiconductor manufacturing. Also, the limitations of this paper cannot integrate diagnosis information based on online data.

Furthermore, a number of interesting directions for further research can be followed based on the ideas proposed in this study. For instance, the newly proposed strategy can be extended to address predictive maintenance problems with a high degree of flexibility by considering prognostics and diagnostics information.

\section{Data Availability}

The data used to support the findings of this study are included within the article.

\section{Conflicts of Interest}

The author declares that there are no conflicts of interest.

\section{Acknowledgments}

This work was supported by University of Shanghai for Science and Technology.

\section{References}

[1] L. Xiao, S. Song, X. Chen, and D. W. Coit, "Joint optimization of production scheduling and machine group preventive maintenance," Reliability Engineering \& System Safety, vol. 146, pp. 68-78, 2016.

[2] C. Javier, M. P. Jose, and A. G. Jose, "An application of dynamic bayesian networks to condition monitoring and fault prediction in a sensored system: a case study," International Journal of Computational Intelligence System, vol. 10, no. 1, pp. 176-195, 2017.

[3] S. Heath and K. D. Yoho, "Using simulation to illuminate process improvement opportunities in a U.S. naval aircraft overhaul facility," Production Planning \& Control, vol. 28, no. 14, pp. 1152-1164, 2017.
[4] Q. Liu and W. Lv, "Multi-component manufacturing system maintenance scheduling based on degradation information using genetic algorithm," Industrial Management \& Data Systems, vol. 115, no. 8, 2015.

[5] Q. Liu, M. Dong, F. F. Chen, W. Lv, and C. Ye, "Singlemachine-based joint optimization of predictive maintenance planning and production scheduling," Robotics and Computer-Integrated Manufacturing, vol. 55, pp. 173-182, 2019.

[6] S. M. Wagner and R. O. Walton, "Additive manufacturing's impact and future in the aviation industry," Production Planning \& Control, vol. 27, no. 13, pp. 1124-1130, 2016.

[7] A. J. Guillén, A. Crespo, M. Macchi, and J. Gómez, "On the role of Prognostics and Health Management in advanced maintenance systems," Production Planning \& Control, vol. 27, no. 12, pp. 991-1004, 2016.

[8] L. Yang, Y. Zhao, R. Peng, and X. Ma, "Hybrid preventive maintenance of competing failures under random environment," Reliability Engineering \& System Safety, vol. 174, pp. 130-140, 2018.

[9] M. Zhang, H. Wang, Z. Cui, and J. Chen, "Hybrid multiobjective cuckoo search with dynamical local search," Memetic Computing, vol. 10, no. 2, pp. 199-208, 2018.

[10] W. Liao, M. Chen, and X. Yang, "Joint optimization of preventive maintenance and production scheduling for parallel machines system," Journal of Intelligent and Fuzzy Systems, vol. 32, no. 1, pp. 913-923, 2017.

[11] Q. Liu, M. Dong, W. Lv, and C. Ye, "Manufacturing system maintenance based on dynamic programming model with prognostics information," Journal of Intelligent Manufacturing, vol. 30, no. 3, pp. 1155-1173, 2019.

[12] C. Drent, S. Kapodistria, and J. A. C. Resing, "Conditionbased maintenance policies under imperfect maintenance at scheduled and unscheduled opportunities," Queueing Systems, vol. 93, no. 7, pp. 269-308, 2019.

[13] E. Pan, W. Liao, and L. Xi, "Single-machine-based production scheduling model integrated preventive maintenance planning," International Journal of Advanced Manufacturing Technology, vol. 50, no. 1-4, pp. 365-375, 2010.

[14] M.-C. Fitouhi and M. Nourelfath, "Integrating noncyclical preventive maintenance scheduling and production planning for a single machine," International Journal of Production Economics, vol. 136, no. 2, pp. 344-351, 2012.

[15] E. Pan, W. Liao, and L. Xi, "A single machine-based scheduling optimisation model integrated with preventive maintenance policy for maximising the availability," International Journal of Industrial and Systems Engineering, vol. 10, no. 4, pp. 451-469, 2012.

[16] S. Wang and M. Liu, "A branch and bound algorithm for single-machine production scheduling integrated with preventive maintenance planning," International Journal of Production Research, vol. 51, no. 3, pp. 847-868, 2013.

[17] C. S. Wong, F. T. S. Chan, and S. H. Chung, "A joint production scheduling approach considering multiple resources and preventive maintenance tasks," International Journal of Production Research, vol. 51, no. 3, pp. 883-896, 2013.

[18] X. Chen, L. Xiao, and X. Zhang, "A production scheduling problem considering random failure and imperfect preventive maintenance," Proceedings of the Institution of Mechanical Engineers Part O Journal of Risk \& Reliability, vol. 229, no. 1, pp. 1-10, 2014.

[19] W. Cui, Z. Lu, and E. Pan, "Integration research of production scheduling and equipment maintenance based on multi-objective optimization," Computer Integrated Manufacturing System, vol. 6, no. 17, pp. 1398-1404, 2014. 
[20] S. Mirabedini and H. Iranmanesh, "A scheduling model for serial jobs on parallel machines with different preventive maintenance (PM)," International Journal of Advanced Manufacturing Technology, vol. 70, no. 9-12, pp. 1579-1589, 2014.

[21] X. Liu, W. Wang, and R. Peng, "An integrated production, inventory and preventive maintenance model for a multiproduct production system," Reliability Engineering \& System Safety, vol. 137, pp. 76-86, 2015.

[22] A. Berrichi, F. Yalaoui, L. Amodeo, and M. Mezghiche, "BiObjective Ant Colony Optimization approach to optimize production and maintenance scheduling," Computers \& Operations Research, vol. 37, no. 9, pp. 1584-1596, 2010.

[23] E. Moradi, S. M. T. Fatemi Ghomi, and M. Zandieh, "Biobjective optimization research on integrated fixed time interval preventive maintenance and production for scheduling flexible job-shop problem," Expert Systems with Applications, vol. 38, no. 6, pp. 7169-7178, 2011.

[24] X. Zhou, Z. Lu, and L. Xi, "Preventive maintenance optimization for a multi-component system under changing job shop schedule," Reliability Engineering \& System Safety, vol. 101, pp. 14-20, 2012.

[25] F. Huang, Z. Lu, and W. Cui, "Joint optimization of production scheduling and maintenance plan to parallel machine system," Industrial Engineering \& Management, vol. 18, no. 4, pp. 49-55, 2013.

[26] M.-C. Fitouhi and M. Nourelfath, "Integrating noncyclical preventive maintenance scheduling and production planning for multi-state systems," Reliability Engineering \& System Safety, vol. 121, pp. 175-186, 2014.

[27] M. Aramon Bajestani, D. Banjevic, and J. C. Beck, "Integrated maintenance planning and production scheduling with Markovian deteriorating machine conditions," International Journal of Production Research, vol. 52, no. 24, pp. 7377-7400, 2014.

[28] M. Pajk, "Genetic-Fuzzy system of power units maintenance schedules generation," Journal of Intelligent and Fuzzy Systems, vol. 28, no. 4, pp. 1577-1589, 2015.

[29] T. Wu, X. Ma, L. Yang, and Y. Zhao, "Proactive maintenance scheduling in consideration of imperfect repairs and production wait time," Journal of Manufacturing Systems, vol. 53, pp. 183-194, 2019.

[30] F. Chang, G. Zhou, C. Zhang, Z. Xiao, and C. Wang, "A service-oriented dynamic multi-level maintenance grouping strategy based on prediction information of multi-component systems," Journal of Manufacturing Systems, vol. 53, pp. 4961, 2019.

[31] T. Yan, Y. G. Lei, B. Wang, T. Y. Han, X. S. Si, and N. P. Li, "Joint maintenance and spare parts inventory optimization for multi-unit systems considering imperfect maintenance actions," Reliability Engineering \& System Safety, vol. 202, 2020.

[32] M. Celen and D. Djurdjanovic, "Integrated maintenance and operations decision making with imperfect degradation state observations," Journal of Manufacturing Systems, vol. 55, pp. 302-316, 2020.

[33] O. Wu, G. Dalle Ave, I. Harjunkoski, and L. Imsland, "A rolling horizon approach for scheduling of multiproduct batch production and maintenance using generalized disjunctive programming models," Computers \& Chemical Engineering, vol. 148, Article ID 107268, 2021.

[34] X. Li, "Research on optimization of setup time for product in the multi-product production system," Microprocessors and Microsystems, vol. 80, Article ID 103618, 2021.
[35] T. Xia, Y. Dong, E. Pan, M. Zheng, H. Wang, and L. Xi, "Fleetlevel opportunistic maintenance for large-scale wind farms integrating real-time prognostic updating," Renewable Energy, vol. 163, pp. 1444-1454, 2021.

[36] Q. Liu, M. Dong, and Y. Peng, "A novel method for online health prognosis of equipment based on hidden semi-markov model using sequential Monte Carlo methods," Mechanical Systems and Signal Processing, vol. 32, no. 2, pp. 331-348, 2012.

[37] C. L. Melchor-Hernández, F. Rivas-Dávalos, S. Maximov, V. Coria, and E. L. Moreno-Goytia, "An analytical method to estimate the Weibull parameters for assessing the mean life of power equipment," International Journal of Electrical Power \& Energy Systems, vol. 64, pp. 1081-1087, 2015. 